\title{
Symmetric and asymmetric states in a mesoscopic superconducting wire in the voltage-driven regime
}

\author{
D. Y. Vodolazov ${ }^{1,2, *}$ and F. M. Peeters ${ }^{2, \dagger}$ \\ ${ }^{1}$ Institute for Physics of Microstructures, Russian Academy of Sciences, 603950, Nizhny Novgorod GSP-105, Russia \\ ${ }^{2}$ Departement Fysica, Universiteit Antwerpen (CGB), Groenenborgerlaan 171, B-2020 Antwerpen, Belgium \\ (Received 23 October 2006; revised manuscript received 15 January 2007; published 27 March 2007)
}

\begin{abstract}
The response of a mesoscopic homogeneous superconducting wire, connected with bulk normal metal reservoirs, is investigated theoretically as a function of the applied voltage. We found both symmetric and asymmetric states which are characterized by a stationary symmetric and asymmetric distribution of the order parameter with respect to the center of the wire. For wires with length $L$ of about the relaxation length of the nonequilibrium quasiparticle distribution function $\overline{L_{E}}$, the asymmetric superconducting state survives up to higher values of the voltage than the symmetric one and may exist both in the voltage- and the current-driven regimes.
\end{abstract}

DOI: 10.1103/PhysRevB.75.104515

PACS number(s): 74.25.Op, 74.20.De, 73.23.-b

In the present paper, we study the voltage response of a dirty superconducting wire (whose mean free path $\ell$ is smaller than the coherence length at zero temperature $\xi_{0}$ ) that is connected to large normal metal reservoirs with no tunnel barriers. Recently, it was theoretically found that the resistance of such a system is a highly nonlinear function of the applied voltage. ${ }^{1}$ The reason is that the applied voltage strongly modifies the quasiparticle distribution function $f(E)$, especially its odd part $f_{L}(E)=f(-E)-f(E)$, which leads to a suppression of the order parameter. ${ }^{1}$ The larger the applied voltage the more pronounced the suppression of the order parameter and hence the larger the resistance of the wire. Actually, it is this mechanism that leads to the appearance of negative differential resistance in the current-voltage characteristics of a superconducting wire in the voltage-driven regime (see Fig. 2 in Ref. 1).

The authors of Ref. 1 considered the case where $f(E, x)$ is symmetrical with respect to the center of the wire (it thus results in a symmetrical suppression of the order parameter from both ends of the wire), but in principle this restriction is not necessarily valid and $f(E, x)$ may also be asymmetric, as we will find in this paper.

A simple example of such a situation is a mesoscopic normal metal wire, half of which has a resistance larger than the other half. By applying a voltage to such a sample, the voltage drop in the high resistance part will be larger than in the low resistance part (we assume that $R_{\text {left }}>R_{\text {right }}$ ) in order to satisfy the continuity of the current along the wire, i.e., $I(x)=$ const. Thus, we have a larger voltage on the left side than on the right one, i.e., $|V(-L / 2)|>|V(L / 2)|$, and the quasiparticle distribution function will be asymmetric.

In a superconducting homogeneous wire, such a situation can be realized if the superconductivity near one of the ends of the wire becomes more strongly suppressed than at the other end at some moment in time [due to fluctuations or due to the special geometry of the sample (see Fig. 3 and discussion at the end of this paper)]. The resistance of that end will be larger than the other one and an asymmetrical voltage drop appears in the wire. Because the deviation of $f_{L}(E, x)$ from equilibrium is stronger at the end with higher voltage $V$, a larger suppression of the order parameter should be found here. Thus, there is some kind of positive feedback and therefore there exists a chance that such an asymmetric state can be stabilized in time. The aim of our paper is to study whether or not such a state can be a stable one.

We use a quasiclassical approach to calculate the nonequilibrium properties of a superconducting wire in the dirty limit and restrict ourselves to temperatures near $T_{c}$. This allows us to use the Usadel equation ${ }^{2}$ for the normal $\alpha(E)$ $=\cos \Theta$ and anomalous $\beta(E)=\sin \Theta$ quasiclassical Green functions, ${ }^{3-5}$

$$
\frac{d^{2} \Theta}{d x^{2}}+\left[\left(2 i E-\frac{1}{L_{E}^{2}}\right)-(\nabla \phi)^{2} \cos \Theta\right] \sin \Theta+2|\Delta| \cos \Theta=0 .
$$

In the same limit, the diffusive-type equation for the space dependence of the transverse (even in energy) $f_{T}(E)=1$ $-f(E)-f(-E)$ and longitudinal (odd in energy) $f_{L}(E)=f($ $-E)-f(E)$ parts of the quasiparticle distribution function $2 f(E)=1-f_{L}(E)-f_{T}(E)$ are given by

$$
\begin{aligned}
& \nabla\left[\left(N_{1}^{2}-R_{2}^{2}\right) \nabla f_{L}\right]+2 N_{2} R_{2} \nabla \phi \nabla f_{T}-\frac{N_{1}}{L_{E}^{2}}\left(f_{L}-f_{L}^{0}\right)=0, \\
& \nabla\left[\left(N_{1}^{2}+N_{2}^{2}\right) \nabla f_{T}\right]+2 N_{2} R_{2} \nabla \phi \nabla f_{L}-\frac{N_{1}}{L_{E}^{2}}\left(f_{T}-\varphi \frac{\partial f_{L}^{0}}{\partial E}\right) \\
& \quad-2 N_{2}|\Delta| f_{T}=0 .
\end{aligned}
$$

Here, $\phi$ is the phase of the order parameter $\Delta=|\Delta| e^{i \phi}, \varphi$ is an electrostatic potential, $N_{1}(E)+i R_{1}(E)=\cos \Theta(E), \quad N_{2}(E)$ $+i R_{2}(E)=\sin \Theta(E)$, and $f_{L}^{0}(E)=\tanh (E / 2 T)$ is the odd part of the equilibrium Fermi-Dirac distribution function of the quasiparticles. The dimensionless length $L_{E}=\sqrt{D \tau_{E}} / \xi_{0}$ $=\sqrt{\tau_{E} \Delta_{0} / \hbar}$ defines the range over which the nonequilibrium distribution of the quasiparticles may exist in the sample $\left[\tau_{E}\right.$ is the finite relaxation time of the nonequilibrium quasiparticle distribution function $f(E)$ due to inelastic electronphonon interaction, $D$ is the diffusion constant].

Within the same approach, we have the rather simple self- 
consistent stationary equation for the order parameter $\Delta$,

$$
a_{1} \frac{d^{2} \Delta}{d x^{2}}+\left(1-a_{2}|\Delta|^{2}+\Psi_{1}+i \Psi_{2}\right) \Delta=0,
$$

which is analogous to the Ginzburg-Landau equation but with the additional terms $\Psi_{1}=\int_{0}^{\infty} R_{2}\left(f_{L}-f_{L}^{0}\right) d E /|\Delta|$ and $\Psi_{2}$ $=\int_{0}^{\infty} N_{2} f_{T} d E /|\Delta|$. Because we are interested in a stationary solution, we removed all time-dependent terms in Eq. (2) which are present in the original equations. ${ }^{3-5}$

In Eqs. (1)-(3), the order parameter $\Delta$ is scaled by the zero-temperature value of the order parameter $\Delta_{0}$ $\simeq 1.76 k_{B} T_{c}$ (in weak coupling limit), distance is in units of the zero temperature coherence length $\xi_{0}=\sqrt{\hbar D / \Delta_{0}}$, and temperature is in units of the critical temperature $T_{c}$. Because of this choice of scaling the numerical coefficients in Eq. (3) are $a_{1} \simeq 0.69$ and $a_{2} \simeq 0.33$. The current is scaled in units of $j_{0}$ $=\Delta_{0} /\left(\xi_{0} \rho_{n} e\right)$ and the electrostatic potential is in units of $\varphi_{0}$ $=\Delta_{0} / e\left(\rho_{n}\right.$ is the normal state resistivity and $e$ is the electric charge).

Through the solution of Eqs. (1)-(3), the current in the system can be found using the following equation:

$$
j=2 a_{1}|\Delta|^{2} \nabla \phi+\int_{0}^{\infty}\left[\left(N_{1}^{2}+N_{2}^{2}\right) \nabla f_{T}+2 N_{2} R_{2} f_{L} \nabla \phi\right] d E .
$$

The first term in Eq. (4) may be identified as the superconducting current and the second one as the normal one. Assuming that the effect of the free charges is negligible in the superconductors, the electrostatic potential is determined by the following expression:

$$
\varphi=\int_{0}^{\infty} N_{1} f_{T} d E
$$

We used the following boundary conditions for Eqs. (1)-(3):

$$
\begin{gathered}
\Theta( \pm L / 2)=\Delta( \pm L / 2)=0 \\
f_{L}( \pm L / 2)=\frac{1}{2}\left[\tanh \frac{E+V( \pm L / 2)}{2 T}+\tanh \frac{E-V( \pm L / 2)}{2 T}\right]
\end{gathered}
$$

and

$$
f_{T}( \pm L / 2)=\frac{1}{2}\left[\tanh \frac{E+V( \pm L / 2)}{2 T}-\tanh \frac{E-V( \pm L / 2)}{2 T}\right]
$$

which models the situation where there is a direct electrical contact of the superconducting wire of length $L$ with large normal metal reservoirs at an applied voltage $V( \pm L / 2)$.

The validity of Eqs. (1)-(3) is restricted to the temperature interval $\Delta(T) / k_{B} T_{c} \ll 1,{ }^{5}$ which roughly corresponds to the condition $0.9<T / T_{c}<1$.

The system of Eqs. (1)-(3) was numerically solved using an iterative procedure. First, Eq. (3) was solved by the Euler method [we add a term $\partial \Delta / \partial t$ in the right-hand side of Eq. (3)] until $|\Delta|$ became time independent. Then, we solved Eqs. (1) and (2) from which we obtained new potentials $\Psi_{1}$ and $\Psi_{2}$ which were then inserted in Eq. (3). This iteration process was continued until convergence was reached. In some voltage interval, no convergence was reached and we identify it as the absence of stationary solutions to Eqs. (1)-(3).

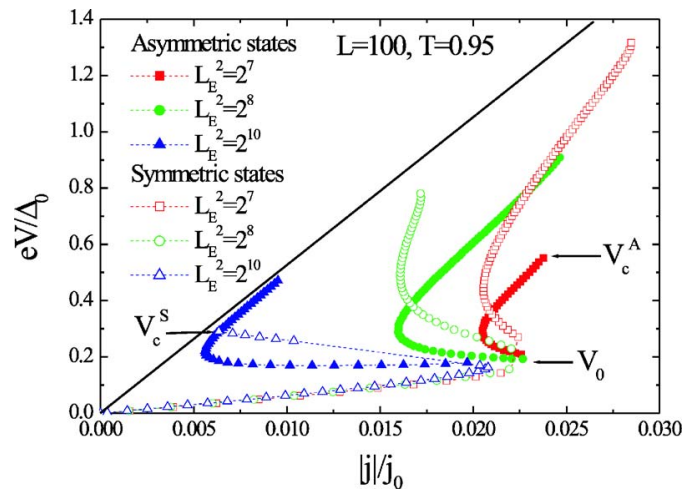

FIG. 1. (Color online) Current-voltage characteristics of the symmetric (empty symbols) and asymmetric (filled symbols) states. Solid curve shows the normal state. Dashed curves mark regions of absence of the stationary symmetric states. The stationary asymmetric state does not exist for $V<V_{0}$-voltage where the negativedifferential resistance appears for the symmetric state. The critical voltages for some of the curves are indicated.

Thus, our numerical procedure automatically checks the stability of the found stationary symmetric and asymmetric states.

Studying the symmetric states (applying symmetric boundary conditions $V( \pm L / 2)=\mp V$ ) for short $\xi \ll L \lesssim L_{E}$ wires, we obtained qualitatively the same results, as in Ref. 1. Namely, the order parameter is influenced by a nonequilibrium $f_{L}$ along the whole wire and at $V \sim \Delta$, it starts to decay steeply. This results in the appearance of a pronounced part with negative differential resistance $d V / d I$ in the $I V$ characteristic (see Fig. 2 in Ref. 1 and Fig. 1 for $L_{E}^{2}=2^{10}$ ) for $V>V_{0}$. The resistance of the wire effectively increases with the suppression of the order parameter and the total current decays. No stationary solutions to Eqs. (1)-(3) were found for samples with lengths larger than approximately $10 \xi(T)$ at $V \sim V_{0}$ and temperatures not far from $T_{c}$.

For long wires $L_{E} \gg L \gg \xi$, we found an $\mathrm{S}$ shape of the current voltage characteristic (see Fig. 1) which we can understand as follows. At $V=V_{0}$, due to the suppression of $|\Delta|$ near the edges by nonequilibrium $f_{L}$, the order parameter decays and the current decreases with increasing voltage. When the region of suppression of $|\Delta|$ increases up to about $L_{E}$, the effect of the nonequilibrium $f_{L}$ becomes less pronounced, $|\Delta|$ decreases much slower with increasing voltage, and the current starts to increase again. At some moment, it reaches a value close to the depairing current density in the center of the wire and the state with a nonequilibrium and a stationary distribution of the order parameter becomes unstable. ${ }^{6}$ Phase slip centers should appear in the wire. This is observed as the absence of a stationary solution to Eqs. (1)-(3) in some range of applied voltage (this is indicated by the dashed lines in Fig. 1). With further increasing voltage the superconducting region decreases (see Fig. 2 for symmetric case), and we find again a stationary solution. The above mechanism leads to an $\mathrm{S}$ shape for the $I V$ characteristics for long wires $L \gg L_{E} \gg \xi(T)$.

To find the asymmetric superconducting states in our homogeneous system, we use the asymmetric boundary conditions $V( \pm L / 2)=\mp V+\Delta V$. The potential constant shift $\Delta V$ is 


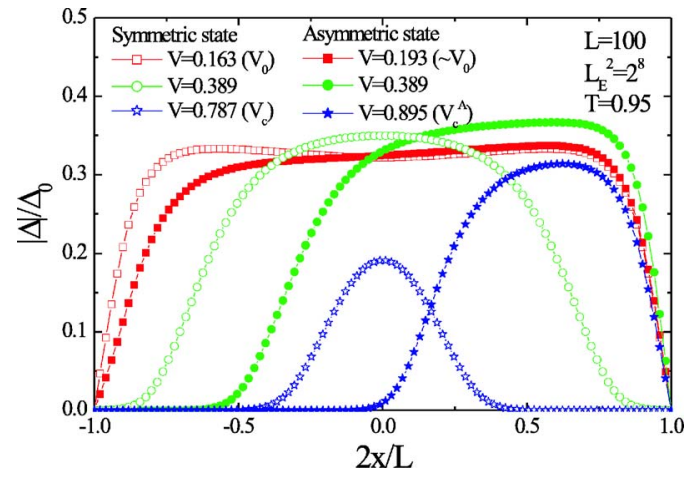

FIG. 2. (Color online) Order parameter distribution for asymmetric and symmetric states.

determined from the condition of constant current along the wire. It increases from zero (at $V \simeq V_{0}$ ) to a finite value (at $V_{c}^{a}$-critical voltage of the asymmetric state). No asymmetric states were found for $V<V_{0}$. In Fig. 1, we show typical $I V$ characteristics and in Fig. 2, we present the corresponding distribution of the order parameter for symmetric and asymmetric states for the same values of the voltage. As expected, the order parameter is more suppressed on the side of the wire with maximal absolute value of the voltage. For example, if we change the sign of $\Delta V$, the order parameter distribution in Fig. 2 will be symmetrically reflected with respect to $x=0$.

It is interesting to note that such an asymmetric state may exist up to larger values of the voltage when $L \gtrsim L_{E} \gg \xi$, and even for larger values of the current, than the symmetric state (the latter property is realized for lengths $L \sim L_{E}$ for which the $I V$ characteristic changes slope from a negative to a positive one for the symmetric state when $\left.V>V_{0}\right)$. Another interesting property is the existence of a stationary asymmetric state for voltages when the stationary symmetric state is absent (see Fig. 1).

The range of the existence of the stationary asymmetric state is rather small in comparison to the symmetric state when the length of the wire is much larger than $L_{E}$. It exists only at voltages near $V_{0}$ (see Fig. 1 for small ratio $L_{E} / L$ ).

We did not find any stationary asymmetric states for wires with $L_{E} \sim \xi(T)$. For small values $L_{E} \ll \xi(T)$, the asymmetric state is nearly the same as the symmetric one but shifted with respect to the point $x=0$ and the $I V$ characteristics are found to be nearly identical for both symmetric and asymmetric states. This is explained by the negligible effect of $f_{L}$ on the value of the order parameter in this limit.

We should stress that the existence of the asymmetric state is not connected with the finite relaxation length $L_{E}$. We checked numerically the presence of such a state for superconducting wires with $L_{E}=\infty$. Because our results for the symmetric state are similar to the findings of Ref. 1, we expect that the asymmetric state in superconducting wire should exist at low temperatures.

Experimentally, the asymmetric state may be realized by adding additional contacts to the superconducting wire with control current close to the ends of the wire (see Fig. 3). It is better to contact the wire with the reservoirs made from the same material and to apply a strong enough magnetic field to

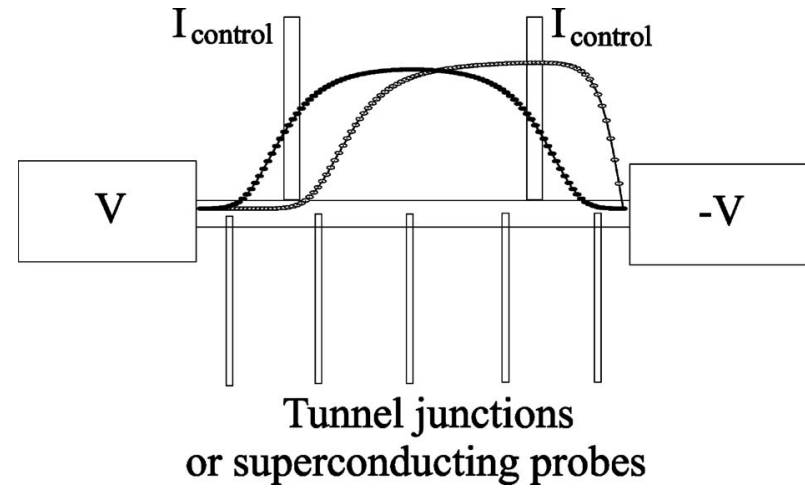

FIG. 3. Proposed experimental setup to measure transitions between symmetric and asymmetric states and the current-voltage characteristic of both states. The curves with empty and filled symbols show the order parameter distribution for a wire with parameters as in Fig. 2 and $V=0.389$.

suppress the superconductivity in the reservoirs (such a procedure provides good contacts with normal reservoirs). Applying a strong enough current to only one of the current contacts, we locally destroy superconductivity and force an asymmetric distribution of the order parameter. After switching off the control current, the asymmetric distribution should be stable (for a proper choice of the working point at the $I V$ characteristic). To return to the symmetric state, we should apply identical control current at both current contacts. We need a series of tunnel junctions along the wire to measure locally the strength of the order parameter. Alternatively, one can use superconducting probes to distinguish the part of the superconductor which is in the normal or superconducting state. When in the normal state, the superconducting probe measures the electrostatic potential.

Any real sample has intrinsic inhomogeneities. In Fig. 4, we present $I V$ characteristic of the sample with weak inhomogeneities on the edges. Namely, we supposed that the right and left ends of the wire with lengths $10 \xi_{0}$ and $13 \xi_{0}$, respectively, have a critical temperature that is locally

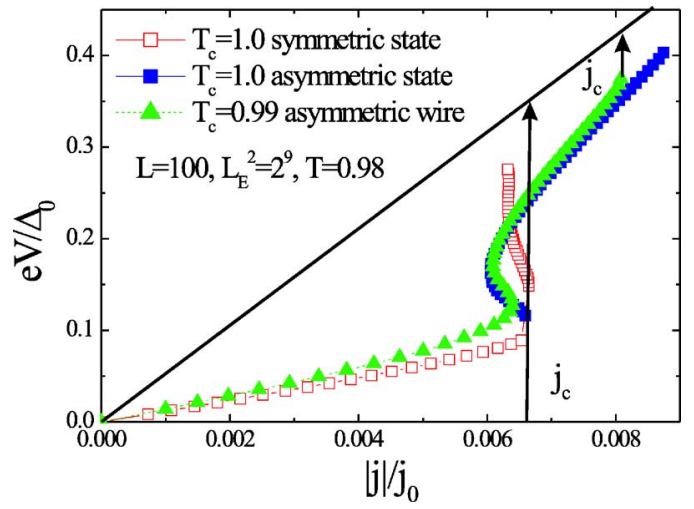

FIG. 4. (Color online) Current-voltage characteristics of the homogeneous wire (solid and black squares) and wire with intrinsic asymmetry in physical properties (gray triangles). For the inhomogeneous wire, we took the critical temperature at the left and right ends of the sample with lengths $10 \xi_{0}$ and $13 \xi_{0}$, respectively, locally smaller by $1 \%$. 
smaller by $1 \%$ as compared to the rest of the sample. This imposes the intrinsic asymmetry to the sample and favors the formation of the asymmetric states when $V \gtrsim V_{0}$. For the parameters used for Fig. 4, it results in larger values of the critical voltage (in voltage-driven regime) and the critical current (in current-driven regime) for the transition to the normal state.

One may also impose the inhomogeneity by using the effect of a magnetic field. If, for example, one of the ends of the wire is wider than the other one, the magnetic field will suppress the order parameter more strongly in the wider part. Then, the transition from symmetric to asymmetric state may lead to an abrupt "enhancement" of the critical current by the applied magnetic field.

Good candidates to observe the predicted asymmetric state are dirty aluminum and zinc with their relatively large coherence lengths $\left[\xi_{\mathrm{Al}}(0) \sim 0.15 \mu \mathrm{m}\right.$ and $\left.\xi_{\mathrm{Zn}}(0) \sim 0.25 \mu \mathrm{m}\right]$ and $\overline{L_{E}}(\mathrm{Al}) \sim 9 \mu \mathrm{m}$ and $\overline{L_{E}}(\mathrm{Zn}) \sim 35 \mu \mathrm{m}\left[L_{E}(\mathrm{Al}) \sim 60\right.$ and $\left.L_{E}(\mathrm{Zn}) \sim 140\right]$. The strictest restriction in order to observe these effects is that there should only be small heating of the sample. Heating will initiate the transition of the sample to the normal state and hide the predicted effects.

For other low-temperature superconductors $(\mathrm{Nb}, \mathrm{Pb}, \mathrm{In}$, and Sn), we have $L_{E}<10$ (we used data for $\tau_{E}$ at $T \sim T_{c}$ from Ref. 7). It means that at $T \geqslant 0.9$, the coherence length is comparable with $L_{E}$. Under these conditions, we did not find the asymmetric state.
To conclude, we theoretically found that for a superconducting wire connected with normal reservoirs, there may be three stationary states at a given value of the applied voltage difference $2 V>2 V_{0}$ - one symmetric state and two asymmetric states. The latter can be transferred into each other by reflecting with respect to the center of the wire. These three states are characterized by symmetric and asymmetric distributions of the order parameter, respectively. The degeneracy is most pronounced in the limit $L \gtrsim L_{E} \gg \xi$ because the order parameter may vary on distances of the order $L_{E} \gg \xi$ due to the long relaxation length of the odd (in energy) part of the nonequilibrium quasiparticle distribution function and it provides the basis for the appearance of different effects. For example, it leads to an $\mathrm{S}$ behavior of the $I V$ characteristic of the wire being in the symmetric state. In the same limit, the stationary asymmetric state may exist even when the symmetric one only exists as a time-dependent one (phase slip state) (see Fig. 1). Moreover, there exists a critical length of the sample for which a stationary asymmetric state does exist up to a larger value of the current than the stationary symmetric one (see Fig. 1 and case $L_{E}^{2}=2^{8}$ ).

This work was supported by the Flemish Science Foundation (FWO-Vl), the Belgian Science Policy (IUAP), and the ESF-AQDJJ program. D.Y.V. acknowledges support from INTAS Young Scientist program (04-83-3139).
*Electronic address: vodolazov@ipm.sci-nnov.ru

†Electronic address: francois.peeters@ua.ac.be

${ }^{1}$ R. S. Keizer, M. G. Flokstra, J. Aarts, and T. M. Klapwijk, Phys. Rev. Lett. 96, 147002 (2006).

${ }^{2}$ K. D. Usadel, Phys. Rev. Lett. 25, 507 (1970).

${ }^{3}$ A. Schmid and G. Schön, J. Low Temp. Phys. 20, 207 (1975).

${ }^{4}$ L. Kramer and R. J. Watts-Tobin, Phys. Rev. Lett. 40, 1041 (1978).

${ }^{5}$ R. J. Watts-Tobin, Y. Krähenbühl, and L. Kramer, J. Low Temp.
Phys. 42, 459 (1981).

${ }^{6}$ Here, we should mention that for relatively large relaxation length $L_{E}>\xi(T)$, the critical current for the stability of the N-S border is larger than the depairing current density and superconductivity becomes unstable in the center of the wire. The reason is that the superconducting current (not the whole current) destroys superconductivity, but it is small at the N-S border for large $L_{E}$.

${ }^{7}$ M. Stuivinga, J. E. Mooij, and T. M. Klapwijk, J. Low Temp. Phys. 46, 555 (1982). 